\title{
SIMULINK Simulation Implementation of DS/FH Hybrid Spread Spectrum Communication System
}

\author{
Bing MA ${ }^{1, a}$, Ye HE ${ }^{1}$, Hualong MA ${ }^{1}$ and Changren YU \\ ${ }^{1}$ Information and Communications College, National University of Defense Technology, Wuhan, China
}

\begin{abstract}
The basic principle of DS/FH hybrid spread spectrum communication system is introduced. The Simulink simulation software is used to establish the system simulation model, and the dynamic simulation of the system is realized. The anti-jamming performance of the system under tracking interference and blocking interference is analyzed.
\end{abstract}

\section{Introduction}

Direct-sequence spread spectrum and frequency hopping are the most widely used two kinds of spread spectrum technologies. They all have strong anti-interference ability, and the advantages and disadvantages of the two are complementary to a large extent. Combining these two kinds of spread spectrum technologies Get up and avoid weaknesses can improve system performance.DS/FH hybrid communication system combines direct sequence spread spectrum technology with frequency hopping technology and is the most viable anti-jamming communication system at home and abroad. It synthesizes the advantages of low spectral density and random frequency hopping of the direct expansion system, and improves the ability of counterreconnaissance; with the addition of one-dimensional frequency domain segmentation, the network can be networked according to the rule of frequency hopping, which overcomes the disadvantages of the pure directswapping system, which is inconvenient for large-scale networking due to the correlation and near-far effect of pseudo-code sequences, and improves the networking capability; with the addition of direct expansion, some of the multi-path interferences are overcome and the ability of moving through is improved. Studying the DS/FH hybrid communication system will have certain practical significance to the civil communication system and the anti-jamming of military communication.

This article will use Simulink software to carry on the simulation analysis to the DS/FH mixed communication system.

\section{Introduction to the basic principle of DS/FH}

The direct-spread/frequency-hopping spread spectrum system is a function of adding a carrier frequency hopping on the basis of a direct-sequence spread spectrum system, and its basic operation method is

\footnotetext{
a Corresponding author: mabing36067443@163.com
}

direct-sequence spread spectrum. Therefore, the system synchronization is based on the direct sequence synchronization. The following is the basic principle of DS/FH hybrid spread spectrum technology, as shown in Figure 1:

In a DS/FH system, the spread spectrum signal consists of a direct sequence signal with a carrier frequency hopping. It can be seen from the figure that the transmitter of the DS/FH system is a direct superposition of the direct sequence modulator and the frequency hopping carrier. The difference between this hybrid modulator and simple direct sequence modulation is that its carrier is changed according to the frequency hopping pattern, but not fixed like the simple DS modulation. The difference from a pure frequency hopping system is that each time it jumps, the directspread power spectrum must be shifted according to the rules of the frequency hopping pattern, and the minimum interval of the hopping channel must be equal to the pseudo-code rate of the direct sequence.

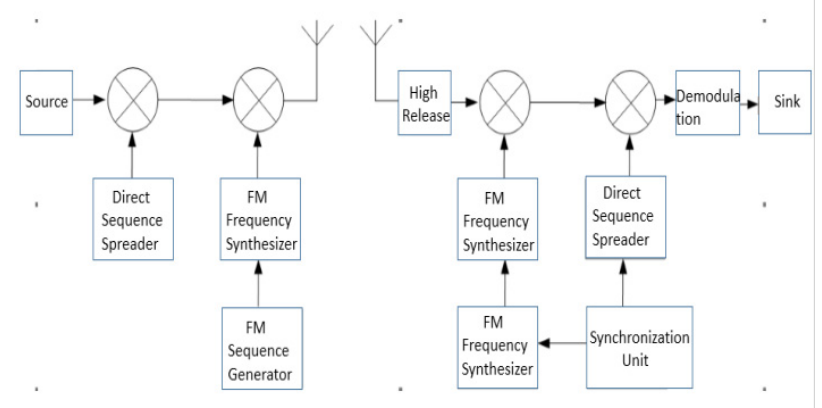

Figure 1. Principle block diagram of DS/FH hybrid spread spectrum system.

The frequency hopping pseudo-random sequence generator in FIG. 1 provides a frequency hopping pattern to the frequency synthesizer, and the direct-spread pseudo-random sequence generator provides a DS spread spectrum modulation sequence to the balanced modulator, so the balanced modulation output is the 
$\mathrm{DS} / \mathrm{FH}$ signal. As long as the frequency of the interference is inconsistent with the frequency hopping frequency, it cannot pass through the IF filter and it cannot affect the useful signal. Even if there is interference and the same frequency of the transmitted signal, the spectrum will be broadened when the directsequence expansion is performed, so that the energy passing through the filter is greatly reduced. Therefore, using this hybrid system can improve the anti-jamming capability and simplify the equipment, reduce the technical difficulty of the extension components, and can achieve the purpose of multiple access.

\section{System Simulation Model Establishment}

\subsection{Overall system model Simulink design}

According to the basic principle of DS/FH hybrid spread spectrum communication system, the Simulink simulation software is used to establish a DS/FH hybrid spread spectrum communication system simulation model, which can better reflect the dynamic working process of DS/FH hybrid library frequency-frequency communication system, observable signal Waveforms, spectral analysis and bit error rate calculations. Figure 2 shows a block diagram of the Simulink model for a DS/FH hybrid spread spectrum communication system.

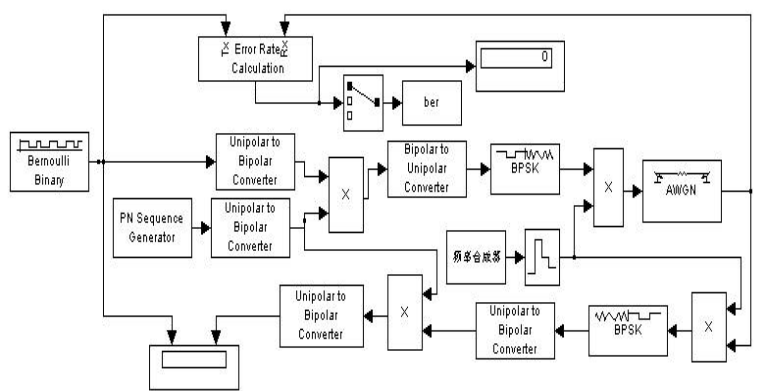

Figure 2. Simulink model block diagram of DS/FH hybrid spread spectrum communication system

Signal source: The Bernoulli binary generator is used as the signal source of the simulation system to generate a binary random signal with an information rate of 1 Kbps.

Spreading and despreading: A PN sequence generator is used to generate a 15-bit $\mathrm{m}$-sequence to spread and despread the source signal.

Modulation and demodulation: use BPSK modulation module for modulation and demodulation. The spreadspectrum wideband signal is modulated by BPSK and demodulated by coherent demodulation after transmission over the channel.

Channel: This article uses an additive white Gaussian noise channel. The module can achieve signal power and signal-to-noise ratio settings.

\subsection{Frequency Hopping Subsystem Frequency Synthesizer Simulink Design}

Figure 3 shows the simulation model of frequency hopping subsystem frequency synthesizer Simulink, The PN sequence generator generates a sampling period of $1 / 400$, An m-sequence with a length of 15 symbols that converts a single-column binary sequence into 4-column binary through a buffer, through a bit-to-integer converter, it becomes a list of hexadecimal integers, after the initial value is set to 4 and the zero order hold circuit, the binary number generated by the pseudo-random sequence generator becomes an integer corresponding to it, feed to the control input of a voltage controlled oscillator, used to generate the output frequency of the control voltage controlled oscillator. Figure 4 shows the frequency synthesizer output waveform.

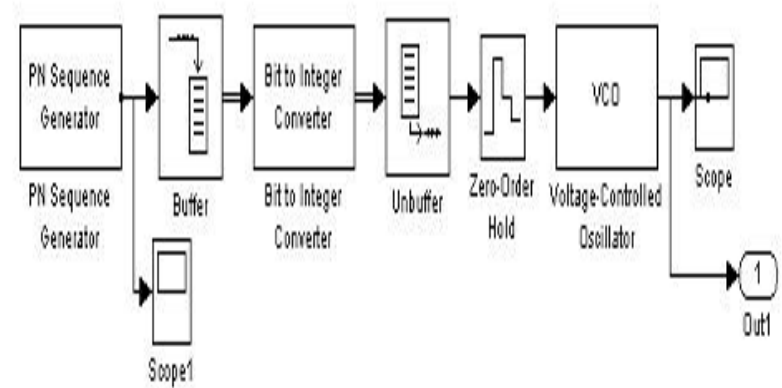

Figure3. Frequency hopping subsystem frequency synthesizer simulink simulation model

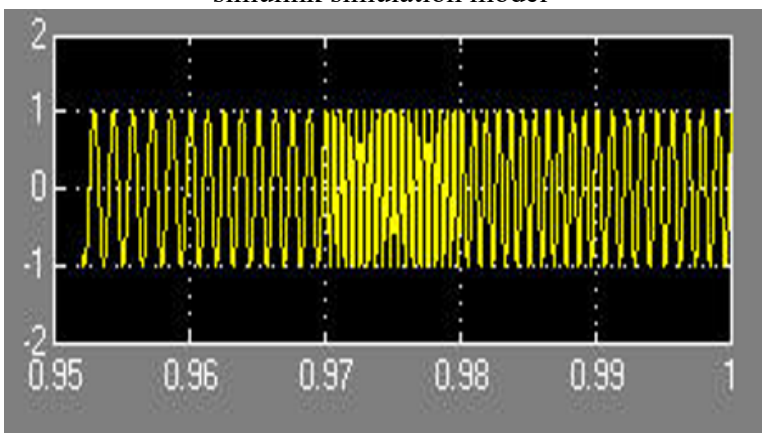

Figure 4. Frequency synthesizer output waveform

\section{Analysis of simulation results}

\subsection{Overall system model Simulink design}

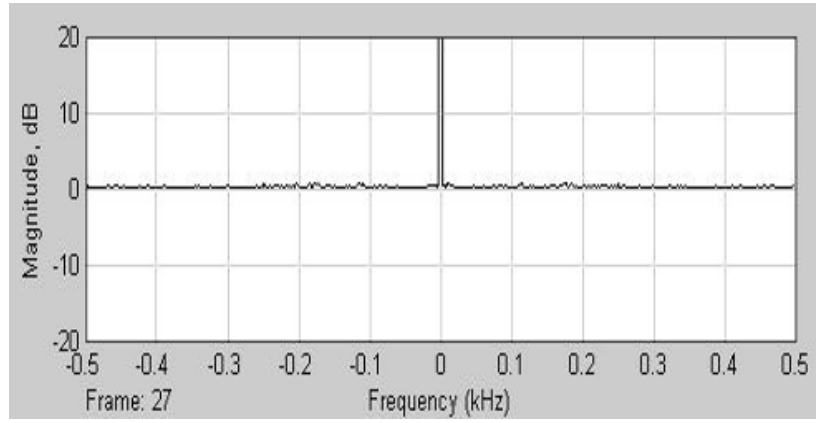

Figure5. The unspread spectrum

Figure 5 shows the spectrum before unspreading. As shown in the figure, before the non-spread spectrum, the signal energy is mostly concentrated in a small range of 
a certain frequency, and it is easy to be discovered and tracked.

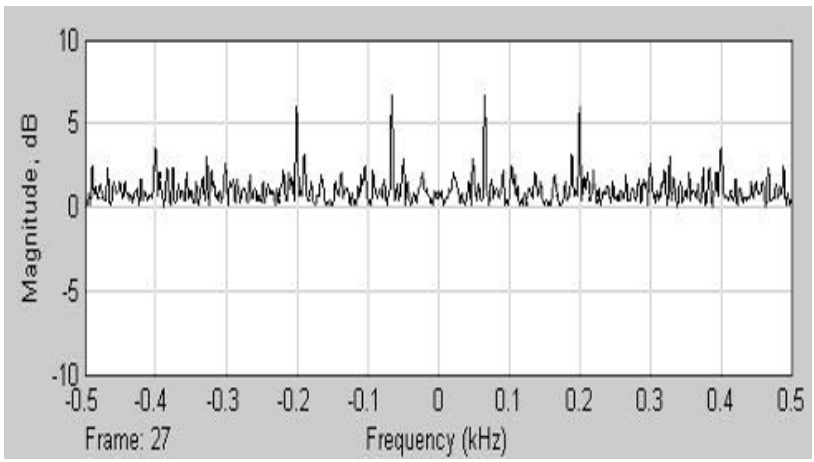

Figure6. The Mixed spectrum spread spectrum

Figure 6 shows the spectrum after mixing spread spectrum. As shown in the figure, under certain power conditions, the bandwidth of the signal is increased, its power spectral density is reduced, and the concealment of the signal and low interception are enhanced.

\subsection{Analysis of simulation results without interference}

Under the simulation conditions of $1 \mathrm{Kbps}$ information rate, $8 \mathrm{~dB}$ signal-to-noise ratio, and $10 \mathrm{~S}$ simulation time, the simulation operation was observed. Figure 7 shows the input and output signals. The waveform of the signal generated by the visible source and the waveform of the output signal of the receiver are basically the same. At the same time observe the BER display module "Display", we can see that the system error rate is 0 , indicating that the Simulink DS / FH hybrid communication system proposed in this article is in line with the non-disturbing, low signal to noise ratio can achieve signal distortion-free transmission Theory.

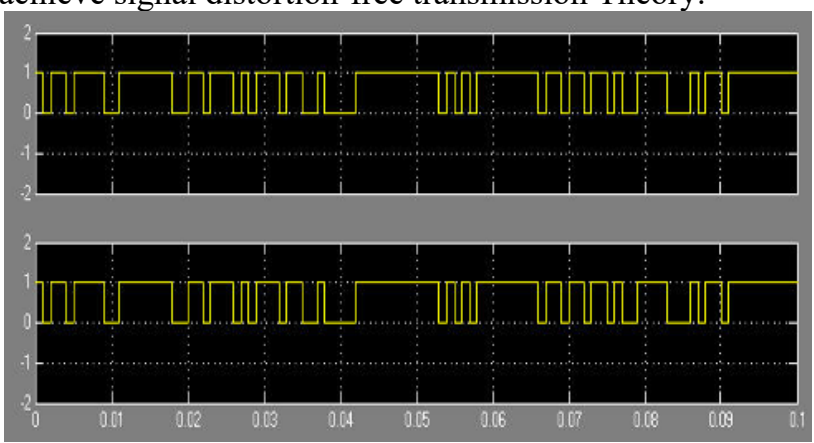

Figure7. System oscilloscope display results

\subsection{Analysis of simulation results under interference}

As can be seen from Figure 8, after adding the system to tracking interference, Since the DS/FH hybrid communication system combines the advantages of frequency hopping and spread spectrum, In particular, inherited frequency hopping can rely on tracking speeds higher than that of tracking jammers to avoid guided tracking interference, relying on the randomness of frequency hopping patterns, and nonlinear avoidance waveform tracking interference.Therefore, the integrated system is better at tracking interference. But for blocking interference, due to limited frequency resources in practical applications, DS/FH hybrid communication system is not effective for improving spread spectrum processing gain. Therefore, the effect of the system against blocking interference is still not ideal.

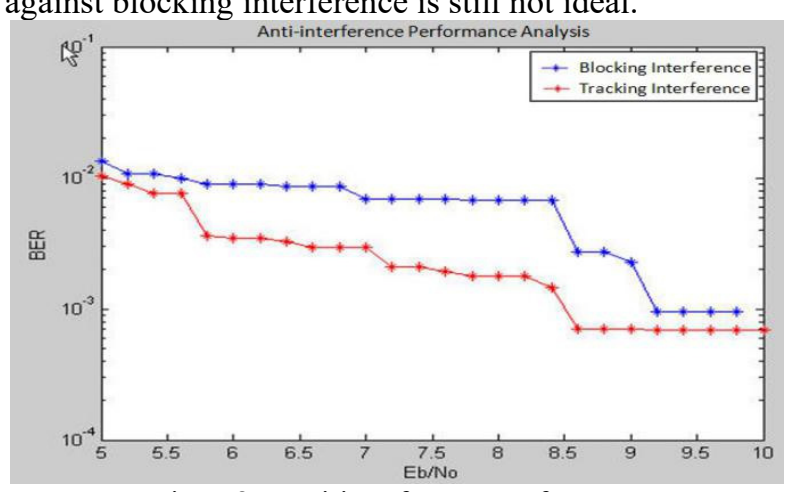

Figure8. Anti-interference performance

\section{Conclusion}

In this paper, the simulation model of Simulink is given, and the simulation of the DS/HFSK spread spectrum system is performed. It is verified that the anticorrelation interference performance is superior to the pure frequency hopping system and the pure directspread system. DS/FH combines the advantages of direct-spreading and frequency hopping, but it also inevitably retains some disadvantages. In practical applications, we can further improve the DS/FH by increasing the length of the pseudo-random code, and gradually improve the anti-jamming capability of the communication.

\section{References}

1. Mao Liangju.Research and Implementation of Deepspace DS/FH Hybrid Spread Spectrum Signal Acquisition Technology[D], University of Electronic Science and Technology,2013.

2. Ding Qiang.Research and Implementation of DS/FH Hybrid Spread Spectrum Receiver[D], Xi'an University of Electronic Science and Technology,2011.

3. Research on Anti-interference and Synchronization of DS/FH Hybrid Spread Spectrum System[D], Shanghai Jiaotong University,2011.

4. Chen Jing.Research on Synchronization and Antiinterference of DS/FH Hybrid Spread Spectrum Measurement and Control Signals[D], University of Electronic Science and Technology,2009.

5. Ao Yu,Yang Jiansheng.Simulation Analysis of Mixed Communication System Based on Simulink[J]. electronics world.2018(07):118.

6. Wang Ling.Simulation of Interference Suppression of Direct Spread Spectrum Communication System Based on Simulink[J].journal of communication university of China.2015,22(6) :21-27. 